International Mathematical Forum, 2, 2007, no. 12, 565 - 574

\title{
A parallel algorithm for the heat equation with derivative boundary conditions
}

\author{
M. Akram \\ University College of Information Technology \\ University of the Punjab, Old Campus \\ Lahore-54000, Pakistan \\ m.akram@pucit.edu.pk
}

\begin{abstract}
In this paper a parallel algorithm is presented for the numerical solution of the heat equation with derivative boundary conditions. The parallel algorithm is developed by approximating the second-order spatial partial derivative by third-order finite-difference approximations and a matrix exponential function by a rational approximation having distinct real poles. Problems are solved using the algorithm and comparisons are made with results from the literature confirming the accuracy of the algorithm.
\end{abstract}

Keywords: Heat equation; Method of lines; Rational approximation; Parallel algorithm

Mathematics Subject Classification: 65M06, 65M20, 68W10

\section{Introduction}

Consider the one-dimensional heat equation

$$
u_{t}=u_{x x}+s(x, t), \quad 0<x<X, \quad t>0
$$

subject to time-dependent boundary conditions on boundary $\partial R$ of the openregion $R$ defined by lines $x=0$ and $x=X$ given by

$$
\begin{aligned}
& u_{x}(0, t)=f_{1}(t), \quad 0<t \leq T, \\
& u_{x}(X, t)=f_{2}(t), \quad 0<t \leq T,
\end{aligned}
$$


with initial condition

$$
u(x, 0)=g(x), \quad 0 \leq x \leq X,
$$

where $f_{1}(t), f_{2}(t), g(x)$ and $s(x, t)$ are known, while the function $u(x, t)$ is to be determined.

The advances in computer technology have provided an impetus to solve physical problems numerically that arise in many branches of continumn physics, such as heat flow, diffusion, wave mechanics, bioengineering, fluid dynamics, elastic vibrations. Since it is essential for modern engineering analysis to have efficient computational schemes to solve complicated mathematical models of physical processes, the authors propose to study numerical techniques for approximating the solutions to various mathematical models related to heat equation with derivative boundary conditions. In this paper the application of the method of lines (MOL) to such problems is considered. The MOL semi-discretization approach will be used to transform the model partial differential equation into a system of first-order linear ordinary differential equations (ODEs). The MOL is a method of solving partial differential equations (PDEs) by discretizing the equation with respect to all but one variable (usually time). The spatial partial derivative is approximated by finite-difference approximations. The solution of the resulting system of first-order ODEs satisfies a recurrence relation which involves a matrix exponential function. Numerical techniques are developed by approximating the exponential matrix function in this recurrence relation. The matrix exponential function is approximated by a rational approximation consisting of three parameters. The parallel algorithm is developed using the resulting approximation.

The demands of both the scientific and the commercial communities for everincreasing computing power led to dramatic improvements in computer architecture. Initial efforts concentrated on achieving high performance on a single processor, but the more recent past has been witness to attempts to harness multiple processors. Multiprocessor systems consist of a number of interconnected processors each of which is capable of performing complex tasks independent of the others. In a sequential algorithm all processes are performed by a single processor turn by turn but in a parallel algorithm independent parts of the program are performed by different processors simultaneously which save a lot of time.

Several sequential numerical methods (implicit as well as explicit) have been proposed in the literature for the solution of this problem $[10,11,12]$. In this paper, we present $O\left(h^{3}+l^{3}\right) L_{0}$-stable parallel algorithm for the numerical solution of the heat equation subject to derivative boundary conditions. The comparison of numerical results clearly demonstrates the computational superiority of this parallel algorithm.

The paper is organized in the following way: the numerical scheme is described 
in Section 2 and the parallel algorithm is presented in Section 3. In Section 4 , the numerical results produced by this scheme are given and summary and concluding remarks are given in Section 5 .

\section{Discretization approach and recurrence re- lation}

The interval $0 \leq x \leq X$ is divided into $N+1$ subintervals each of width $h$, so that $(N+1) h=X$ and the time variable $t$ is discretized in the steps of length $l$. Thus at each time level $t=t_{n}=n l(n=0,1,2, \cdots)$, the open region $R=\{0<x<X\} \times[t>0]$ and its boundary $\partial R$ consisting of lines $x=0$ and $x=X$ and the axis $t=0$ have been superimposed by rectangular mesh with $N$ points within $R$ and open point along each side of $\partial R$. The solution $u(x, t)$ of (1) is sought at each point $(k h, n l)$ in $R \times[t>0]$, where $k=1,2, \cdots, N$ and $n=0,1,2, \cdots$. The solution of an approximating numerical method will be denoted by $U(x, t)$. The space derivative in (1) and boundary conditions will be replaced by the following difference approximations:

$$
\begin{aligned}
\frac{\partial u(x, t)}{\partial x} & \simeq \frac{1}{2 h}\{u(x+h, t)+0 u(x, t)-u(x-h, t)\}, \\
\frac{\partial^{2} u(x, t)}{\partial x^{2}} & \simeq \frac{1}{12 h^{2}}\{11 u(x-h, t)-20 u(x, t)+6 u(x+h, t) \\
& +4 u(x+2 h, t)-u(x+3 h, t)\}, \text { as } h \rightarrow 0 .
\end{aligned}
$$

Note that implementation of (6) requires some additional values for $x=$ $x_{N-1}, x_{N}$. Hence at these points the following third-order approximations will be used:

$$
\begin{aligned}
\frac{\partial^{2} u(x, t)}{\partial x^{2}} & \simeq \frac{1}{12 h^{2}}\{u(x-3 h, t)-6 u(x-2 h, t)+26 u(x-h, t)-40 u(x, t) \\
& +21 u(x+h, t)-2 u(x+2 h, t)\} \\
\frac{\partial^{2} u(x, t)}{\partial x^{2}} & \simeq \frac{1}{12 h^{2}}\{2 u(x-4 h, t)-11 u(x-3 h, t)+24 u(x-2 h, t) \\
& -14 u(x-h, t)-10 u(x, t)-9 u(x+h, t)\}
\end{aligned}
$$

Thus the boundary conditions $u(0, t)$ and $u(X, t)$ can be determined using $(2)$ and (3) with (5) respectively. Applying (1) to all the interior mesh points 
within $R$ at time level $t=n l$ with the space derivative replaced by (6-8) leads to a system of $N$ first-order ODE's of the form

$$
\frac{d \mathbf{U}(t)}{d t}=A \mathbf{U}(t)+\mathbf{v}(t), \quad t>0
$$

with the initial condition

$$
\mathbf{U}(0)=\mathbf{g}
$$

in which the matrix $A$ is of order $N$ and given by

$$
A=\frac{1}{12 h^{2}}\left[\begin{array}{rrrrrrrr}
-20 & 17 & 4 & -1 & & & & 0 \\
11 & -20 & 6 & 4 & -1 & & & \\
& 11 & -20 & 6 & 4 & -1 & & \\
& & \ddots & \ddots & \ddots & \ddots & \ddots & \\
& & & 11 & -20 & 6 & 4 & -1 \\
& & & & 11 & -20 & 6 & 4 \\
0 & & & 2 & -5 & 24 & -31 & 11 \\
& & & & 24 & -23 & -10
\end{array}\right]
$$

In equation (9) the vector $\mathbf{v}(\mathrm{t})$ aries from the use of boundary conditions $u(0, t)$ and $u(X, t)$ in (6-8). Solving (9) subject to (10) gives

$$
\mathbf{U}(t)=\exp (l A) \mathbf{g}+\int_{0}^{t} \exp ((t+l-s) A) \mathbf{v}(s) d s
$$

which satisfies [1] the recurrence relation

$$
\mathbf{U}(t+l)=\exp (l A) \mathbf{U}(t)+\int_{t}^{t+l} \exp ((t+l-s) A) \mathbf{v}(s) d s ; \quad t=0, l, 2 l, \ldots
$$

Following Taj and Twizell [9] third-order accuracy in the temporal component is accomplished by approximating the matrix exponential function $\exp (l A)$ in (13) by

$$
\exp (l A)=G^{-1} N
$$

in which

$$
N=\left(I+\left(1-a_{1}\right) l A+\left(\frac{1}{2}-a_{1}+a_{2}\right) l^{2} A^{2}\right)
$$


and

$$
G=I-a_{1} l A+a_{2} l^{2} A^{2}-\left(\frac{1}{6}-\frac{a_{1}}{2}+a_{2}-a_{3}\right) l^{3} A^{3} .
$$

The denominator of $\exp (l A)$ has distinct real zeros provided that $a_{2}^{2}-3 a_{1} a_{3}>0$ by choosing values $a_{1}=1.308617, a_{2}=0.570502, a_{3}=0.082856$ and $L_{0^{-}}$ stability is introduced in [9]. three aims of $\left(h^{3}+l^{3}\right)$ accuracy, $L_{0}$-stability and use of real arithmetic are met by this choice of the values of $a_{1}$, $a_{2}$ and $a_{3}$. The integral term appearing in (13) is approximated by a quadrature formula of the form

$$
\int_{t}^{t+l} \exp ((t+l-s) A) \mathbf{v}(s) d s \simeq \sum_{j}^{3} W_{j} \mathbf{v}\left(s_{j}\right)
$$

where all $s_{i}(i=1,2,3)$ are different and $W_{1}, W_{2}$ and $W_{3}$ are matrices. It can easily be shown that

- When $\mathbf{v}(s)=[1,1,1, \ldots, 1]^{T}$

$$
W_{1}+W_{2}+W_{3}=M_{1}
$$

where $M_{1}=A^{-1}(\exp (l A)-I)$.

- When $\mathbf{v}(s)=[s, s, s, \ldots, s]^{T}$

$$
s_{1} W_{1}+s_{2} W_{2}+s_{3} W_{3}=M_{2}
$$

where $M_{2}=A^{-1}\left\{t \exp (l A)-(t+l) I+A^{-1}(\exp (l A)-I)\right\}$.

- When $\mathbf{v}(s)=\left[s^{2}, s^{2}, \ldots, s^{2}\right]^{T}$

$$
s_{1}^{2} W_{1}+s_{2}^{2} W_{2}+s_{3}^{2} W_{3}=M_{3}
$$

where $M_{3}=A^{-1}\left[t^{2} \exp (l A)-(t+l)^{2} I+2 A^{-1}\left\{t \exp (l A)-(t+l)+A^{-1}(\exp (l A)-\right.\right.$ $I)\}]$. Taking $s_{1}=t, s_{2}=t+\frac{l}{2}, s_{3}=t+l$ and then solving (17), (18) and (19) simultaneously and replacing by $\exp (l A)$ gives

$$
\begin{aligned}
& W_{1}=\frac{l}{6}\left\{\left(I+\left(4-9 a_{1}+12 a_{2}\right) l A\right\} G^{-1},\right. \\
& W_{2}=\frac{2 l}{3}\left\{\left(I-\left(1-3 a_{1}+6 a_{2}\right) l A\right\} G^{-1},\right.
\end{aligned}
$$

and

$$
W_{3}=\frac{l}{6}\left\{\left(I+\left(3-9 a_{1}+12 a_{2}\right) l A+\left(1-3 a_{1}+6 a_{2}\right) l^{2} A^{2}\right\} G^{-1} .\right.
$$

Hence

$$
\mathbf{U}(t+l)=\exp (l A) \mathbf{U}(t)+W_{1} \mathbf{v}(t)+W_{2} \mathbf{v}\left(t+\frac{l}{2}\right)+W_{3} \mathbf{v}(t+l)
$$

in which $W_{1}, W_{2}$ and $W_{3}$ are given by (20)-(22) respectively. 


\section{Parallel algorithm}

We focused on the construction of a rational approximation with real and distinct poles. So the resulting algorithm readily admits parallelization through partial fraction expansion [2]. An algorithm for implementing (23) discussed in [9]. We represent the parallel algorithm using three different processors in the following form:

Processor 1

(a) Input $l, r_{1}, \mathbf{U}(0), A$

(b) Compute $p_{1}, p_{4}, p_{7}, p_{10}$ and $I-\frac{l}{r_{1}} A$

(c) Decompose $I-\frac{l}{r_{1}} A=L_{1} U_{1}$

(d) Evaluate $\mathbf{v}(t), \mathbf{v}\left(t+\frac{l}{2}\right), \mathbf{v}(t+l)$

(e) use $\mathbf{z}_{1}(t)=\frac{l}{6}\left(p_{4} \mathbf{v}(\mathbf{t})+4 p_{7} \mathbf{v}\left(\mathbf{t}+\frac{\mathbf{l}}{2}\right)+p_{10} \mathbf{v}(t+l)\right)$

(f) Solve $L_{1} U_{1} \mathbf{y}_{1}(t)=p_{1} \mathbf{U}(t)+\mathbf{z}_{1}(t)$

\section{Processor 2}

(a) Input $l, r_{2}, \mathbf{U}(0), A$

(b) Compute $p_{2}, p_{5}, p_{8}, p_{11}$, and $I-\frac{l}{r_{2}} A$

(c) Decompose $I-\frac{l}{r_{2}} A=L_{2} U_{2}$

(d) Evaluate $\mathbf{v}(t), \mathbf{v}\left(t+\frac{l}{2}\right), \mathbf{v}(t+l)$

(e) use $\mathbf{z}_{2}(t)=\frac{l}{6}\left(p_{5} \mathbf{v}(\mathbf{t})+4 p_{8} \mathbf{v}\left(t+\frac{l}{2}\right)+p_{11} \mathbf{v}(t+l)\right)$

(f) Solve $L_{2} U_{2} \mathbf{y}_{2}(t)=p_{2} \mathbf{U}(t)+\mathbf{z}_{2}(t)$

\section{Processor 3}


(a) Input $l, r_{3}, \mathbf{U}(0), A$

(b) Compute $p_{3}, p_{6}, p_{9}, p_{12}, I-\frac{l}{r_{3}} A$

(c) Decompose $I-\frac{l}{r_{3}} A=L_{3} U_{3}$

(d) Evaluate $\mathbf{v}(t), \mathbf{v}\left(t+\frac{l}{2}\right), \mathbf{v}(t+l)$

(e) use $\mathbf{z}_{3}(t)=\frac{l}{6}\left(p_{6} \mathbf{v}(t)+4 p_{9} \mathbf{v}\left(t+\frac{l}{2}\right)+p_{12} \mathbf{v}(t+l)\right)$

(f) Solve $L_{3} U_{3} \mathbf{y}_{3}(t)=p_{3} \mathbf{U}(t)+\mathbf{z}_{3}(t)$

Hence $\mathbf{U}(t+l)=\mathbf{y}_{1}(t)+\mathbf{y}_{2}(t)+\mathbf{y}_{3}(t)$.

GOTO step (d) for next time step.

Implementing the algorithm, Processor 1 generates decomposition of $I-\frac{l}{r_{1}} A$, while Processor 2 generates decomposition of $I-\frac{l}{r_{2}} A$ and Processor 3 generates decomposition of $I-\frac{l}{r_{3}} A$ simultaneously. Parallel computing is the simultaneous execution of the same task (split up and specially adapted) on multiple processors in order to obtain faster results. The idea is based on the fact that the process of solving a problem usually can be divided into smaller tasks, which may be carried out simultaneously with some coordination.

\section{Computational results}

In order to test the behavior of $L_{0}$-stable scheme, three problems from the literature are considered. The parallel algorithm is tested on a sequential computer (Intel. $933 \mathrm{MHz}$, BD815 Glly, 128MB(Kingstung), HDD 20 GB (SeaCate), OS Win2000 Professional, Developer Stuido ) for the solutions of the heat equations.

Problem 1. Consider the heat equation with

$$
\begin{aligned}
s(x, t) & =-2 e^{x-t} \\
g(x) & =e^{x} \\
f_{1}(t) & =e^{-t} \\
f_{2}(t) & =e^{1-t}
\end{aligned}
$$

The analytical solution is $u(x, t)=e^{x-t}$. Using algorithm, this problem is solved for $h=0.125,0.1,0.05,0.025$ with each $l=0.125,0.1,0.05,0.025$. The absolute relative errors $|(u-U) / u|$ at time $t=1.0$ are shown in Table 1 . 


\begin{tabular}{l|cccc}
\hline$h \longrightarrow$ & 0.125 & 0.1 & 0.05 & 0.025 \\
\hline$l=0.125$ & $0.45 \mathrm{D}-4$ & $0.32 \mathrm{D}-4$ & $0.10 \mathrm{D}-4$ & $0.46 \mathrm{D}-5$ \\
$l=0.1$ & $0.34 \mathrm{D}-4$ & $0.11 \mathrm{D}-4$ & $0.65 \mathrm{D}-5$ & $0.31 \mathrm{D}-5$ \\
$l=0.05$ & $0.11 \mathrm{D}-4$ & $0.46 \mathrm{D}-5$ & $0.23 \mathrm{D}-5$ & $0.47 \mathrm{D}-6$ \\
$l=0.025$ & $0.34 \mathrm{D}-5$ & $0.11 \mathrm{D}-5$ & $0.45 \mathrm{D}-6$ & $0.22 \mathrm{D}-6$ \\
\hline
\end{tabular}

Problem 2. Consider the heat equation with

$$
\begin{aligned}
s(x, t) & =x t^{2} \\
g(x) & =\sin (x) \\
f_{1}(t) & =1 \\
f_{2}(t) & =\sin (t)
\end{aligned}
$$

Using the algorithm, this problem is solved for $h=0.125,0.1,0.05,0.025$ with each $l=0.125,0.1,0.05,0.025$. The absolute relative errors $|(u-U) / u|$ at time $t=1.0$ are shown in Table 2 .

\begin{tabular}{l|cccc}
\hline$h \longrightarrow$ & 0.125 & 0.1 & 0.05 & 0.025 \\
\hline$l=0.125$ & $0.74 \mathrm{D}-4$ & $0.24 \mathrm{D}-4$ & $0.13 \mathrm{D}-4$ & $0.10 \mathrm{D}-4$ \\
$l=0.1$ & $0.31 \mathrm{D}-4$ & $0.16 \mathrm{D}-4$ & $0.13 \mathrm{D}-4$ & $0.41 \mathrm{D}-5$ \\
$l=0.05$ & $0.27 \mathrm{D}-4$ & $0.11 \mathrm{D}-4$ & $0.57 \mathrm{D}-5$ & $0.32 \mathrm{D}-5$ \\
$l=0.025$ & $0.11 \mathrm{D}-4$ & $0.22 \mathrm{D}-5$ & $0.15 \mathrm{D}-5$ & $0.12 \mathrm{D}-5$ \\
\hline
\end{tabular}

Problem 3. Consider the heat equation with

$$
\begin{aligned}
s(x, t) & =0 \\
g(x) & =\sin (\pi x) \\
f_{1}(t) & =\pi e^{-\pi^{2} t} \\
f_{2}(t) & =-\pi e^{\pi^{2} t}
\end{aligned}
$$

The problem has analytical solution $u(x, t)=e^{-\pi^{2} t} \sin (\pi x)$. The results of $u(0.5,1.0)$ with different number of time steps are shown in Table 3 . The results obtained using the new scheme developed in this paper are more accurate than those from the scheme of [12]. Note that the new scheme will require less CPU time. It is clear that as far as efficiency is concerned, the scheme introduced in this paper is a better candidate for the model problem. 


\begin{tabular}{l|cc}
\hline Time Steps & {$[12]$} & Parallel Algorithm \\
\hline 10 & 0.001127 & 0.00003415 \\
20 & 0.00828 & 0.00002131 \\
30 & 0.002514 & 0.00002137 \\
40 & 0.003616 & 0.000021164 \\
50 & 0.004299 & 0.00002211 \\
60 & 0.004718 & 0.00003212 \\
70 & 0.004974 & 0.00001281 \\
80 & 0.005130 & 0.00001248 \\
90 & 0.005225 & 0.000011381 \\
100 & 0.005284 & 0.00001121 \\
110 & 0.005319 & 0.00001323 \\
120 & 0.005341 & 0.00001232 \\
130 & 0.005354 & 0.00001121 \\
140 & 0.005362 & 0.00001271 \\
150 & 0.005367 & 0.00001323 \\
\hline
\end{tabular}

\section{Concluding remarks}

In this paper a parallel algorithm was applied to the one-dimensional heat equation with derivative boundary conditions. The algorithm, which may be implemented on a parallel architecture using three processors requires the application of tridiagonal solvers (only). This scheme developed for a parabolic equation subject to Neumann boundary conditions. The second-order spatial derivative was discretized to result in an approximating system of ODEs. The exact solution of this system of first order ODEs satisfies a recurrence relation involving the matrix exponential function. This function is approximated by a rational function possessing real and distinct poles which consequently readily admits a partial fraction expansion thereby allowing the distribution of the work in solving the corresponding linear algebraic systems on concurrent processors. The method developed does not require the use of complex arithmetic and need only real arithmetic in its implementation. This technique worked very well for one-dimensional parabolic equation subject to Neumann boundary conditions. The use of only real arithmetic especially in the threedimensional models can yield large saving in CPU time used. For the model problem considered, the parallel algorithm was found to be about three times faster than the standard implicit finite difference scheme of [12]. A comparison with the standard finite difference schemes for the model problem clearly demonstrates that the new technique is computationally superior. The numerical tests obtained by using the method described in this article give acceptable results and suggests convergence to the exact solution when h goes to zero. 
Acknowledgement: This research work is supported by Punjab University College of Information Technology (PUCIT).

The author is highly thankful to Professor Dr. Shahid S. Siddiqi for his moral support.

\section{References}

[1] J. D. Lambert, Numerical methods for ordinary differential systems: The initial-value problem, John Wiley and Sons, Chichester, 1991.

[2] A. R. Gourlay and J. Morris, The extrapolation of first order methods for parabolic partial differential equations. II, SIAM J. Numer. Anal. 17(5)(1980) 641-655.

[3] D. A. Swayne, Time-dependent boundary and interior forcing in locally onedimensional schemes, SIAM J. Sci. Stat. Comput., 8(1987) 755-767.

[4] J. R. Cannon, Y. Lin and S. Wang, An implicit finite difference scheme for the diffusion equation subject to mass specification, Int.J. Engng. 28(7) (1990) 573-578.

[5] R. Chapko, R. Kress and J. R. Yoon, An inverse boundary value problem for the heat equation: the Neumann condition, Inverse problems 15(1999) 1033-1046.

[6] Z. David and D. Paul, Applied partial differential equations, Dover Publication, Inc., 31 Eash 2nd street, Mineola , N.Y.11501, U.S.A, 2002.

[7] M. Dehghan, Numerical solution of a non-local boundary value problem with Neumann's boundary conditions, 19(1)2002 1-12.

[8] E. H. Twizell, Computational methods for partial differential equations, Ellis Horwood Limited Chichester and John Wiley and Sons, New York ,1984.

[9] M. S. A. Taj and E. H. Twizell, A family of third-order parallel splitting methods for parabolic partial differential equations, Intern. J. Computer Math. 67 (1998) 411-433.

[10] G. E. Forsythe and W. R. Wasow, Finite difference methods for partial differential equations, John Wiley and sons: New York, 1960.

[11] A. Zafarullah, Some stable implicit difference methods for heat equation with derivative boundary condition, The Computer Journal, 14(1971) 309 - 311.

[12] P. Keast and A. R. Mitchell, On the instability of the Crank Nicholson formula under derivative boundary conditions, The Computer Journal, 9(1)(1966) 110114.

Received: September 28, 2005 